\title{
Protective effect of Garcinia against renal oxidative stress and biomarkers induced by high fat and sucrose diet
}

\author{
Kamal A Amin ${ }^{1 *}$, Hamdy H Kamel², Mohamed A Abd Eltawab
}

\begin{abstract}
Background: Obesity became major health problem in the world, the objective of this work was to examine the effect of high sucrose and high fat diet to induce obesity on antioxidant defense system, biochemical changes in blood and tissue of control, non treated and treated groups by administration of Garcinia cambogia, and explore the mechanisms that link obesity with altered renal function

Methods: Rats were fed a standard control diet for 12 week (wk) or a diet containing 65\% high sucrose (HSD) or $35 \%$ fat (HFD) for 8 wk and then HFD group divided into two groups for the following 4 wks. One group was given Garcinia+HFD, the second only high fat, Also the HSD divided into two groups, $1^{\text {st }}$ HSD+Garcinia and $2^{\text {nd }}$ HSD. Blood and renal, mesenteric, Perirenal and epididymal adipose tissues were collected for biochemical assays.

Results: HFD and HSD groups of rats showed a significant increase in feed intake, Body weight (BW) and body mass index (BMI). Also there were significant increases in weights of mesenteric, Perirenal and epididymal adipose tissues in HFD and HSD groups.

HFD and HSD affect the kidney by increasing serum urea and creatinine levels and decreased level of nitric oxide (NO) and increased blood glucose, low density lipoproteins (LDL), triacylglycerol (TG), total cholesterol (TC) and malondialdehyde (MDA). Glucose 6-phosphate dehydrogenase (G6PD) activities were significantly decreased in HFD while there were significant increases in HSD and HSD $+G$ groups $p \leq 0.05$ compared with control. Moreover, renal catalase activities and MDA levels were significantly increased while NO level was lowered. These changes improved by Garcinia that decreased the oxidative stress biomarkers and increased NO level.

There were significant positive correlations among BMI, kidney functions (Creatinine and urea), TG and Oxidative markers (renal MDA and catalase).

Conclusions: Rats fed a diet with HFD or HSD showed, hypertriglyceridemia, increased LDL production, increased oxidative stress and renal alteration. Moreover, suggesting association between lipid peroxidation, obesity and nephropathy, while Garcinia ameliorated the damaging effects of the HFD or HSD and decreased feed intake, MDA level and decreased oxidative stress in renal tissues.
\end{abstract}

\section{Introduction}

Overweight and obesity affected $1.6 \%$ of $2-6$ year olds, $4.9 \%$ of $6-10$ year olds, $14.7 \%$ of $10-14$ year olds, and $13.4 \%$ of $14-18$-year-old children. The prevalence overweight and obesity were 45.3 and $20 \%$ respectively in Egyptian urban men, while they were $28.1 \%$ and $6.0 \%$ respectively in rural men [1].

\footnotetext{
* Correspondence: kaamin10@yahoo.com

'Biochemistry Department, Faculty of Vete. Medicine, Beni-Suef University, Beni-Suef, Egypt

Full list of author information is available at the end of the article
}

Over the past 15 years, there has been an increase in the scientific interest in the impact of high fat and sucrose diet on health. Several experimental studies have been published on the cardiovascular (CV) and hepatic relation to obesity. There was a shortage of data and research about the effect of obesity on renal pathophysiology and biochemical relationship which deserves further investigation.

The pathogenesis of diabetic nephropathy remains far from obvious, partly due to the lack of a suitable animal model that mimics human renal disease in type 2 diabetes.

\section{Biomed Central}


Therefore our aim was to develop rodent model of obesity by HFD and HSD and to demonstrate the biochemical changes and mechanisms that link obesity with altered renal function and its treatment with Garcinia cambogia. Also this study evaluates the contribution of the serum and/or renal $\mathrm{NO}$, oxidative stress and renal function to the renoprotection conferred by the Garcinia in rats.

\section{Materials and methods}

This study was approved by the Committee of Scientific Ethics at Beni Suef University and according to its guidelines.

\section{Material}

1- Diet

three types of diets have been used, control rat chow diet, special HFD (35\%) and special HSD (65\%) for induction of obesity in rats.

a- Normal rat chow diet: It was formed according to Kim et al. [2]. The standard normal rat chow consists of concentrate (350 gm), corn (600 gm), calcium carbonate, dicalcium phosphate, sodium chloride magnesium oxide and vitamins (50 gm).

Standard normal rat diet composed of $65 \%$ CHO $(60 \%$ starch $+5 \%$ sucrose), Fat $5 \%$, crude protein $20 \%$, vitamins and minerals $5 \%$, fibers $5 \%$, metabolic energy of this diet is $2813 \mathrm{Kcal} / \mathrm{Kg}$ with $8 \%$ from fat

b- The high fat diet, composed of 300 gm concentrates, 350 corn, 300 gm beef tallow, 50 gm vitamins, minerals and fibres according to Kim et al. [2] and Amin and Nagy [3]. Calculations of HFD was $20 \%$ crude protein, $35 \%$ fat, $40 \% \mathrm{CHO}$ (starch 35\%, 5\% sucrose) $5 \%$ vitamins and minerals and fibres. Metabolic energy of this diet is $5130 \mathrm{Kcal} / \mathrm{kg} 61 \%$ of this energy from fat.

c- High sucrose diet, consists of 600 gm sucrose, 250 gm concentrate, 100 gm methionine, 50 gm fibers, vitamins and minerals. The chemical analysis of this diet is $20 \%$ crude protein, $5 \%$ fat, $5 \%$ starch, sucrose $65 \%$ and $5 \%$ fibres, vitamins and minerals according to D'Alessandro et al. [4]. Metabolic energy of this diet is $3410 \mathrm{Kcal} / \mathrm{kg}$. $73 \%$ from this energy produced from sucrose.

\section{2- Experimental animals}

The present study carried out on male Wister rat range from 100-130 gm body weight, 8 wks old. They were purchased from the, Hellwan, Cairo, Egypt.

Rats were kept under observation for one wk before onset of experiment to be acclimatized and housed individually in metal cages at room temperature $37^{\circ} \mathrm{C}$, less than 12 hrs light dark cycle in the laboratory of biochemistry in Faculty of Veterinary Medicine-Beni Suef University during the period of the research. Water and special diet were allowed to rats in free manner. Body weights were recorded weekly and food consumption was calculated daily.

\section{3-Drugs for treatment}

Garcinia, drug used by dissolving one capsule in $10 \mathrm{ml}$ D.W each capsule containing $500 \mathrm{mg}$ hydroxy citric acid (HCA) were purchased from the EVA Pharm.

Oral administration of a dose of $50 \mathrm{mg} / \mathrm{rat}$ daily was done according to the method described by Ohia et al. [5]. Handling of the animal was the same for all groups and did not affect weight gain. The drug were given to rat by industrialized stomach tube and given slowly to rat stomach.

\section{Methods}

1-Experimental design and animal grouping

Our experiment continued for $12 \mathrm{wk}$ and divided into two phases (induction period and treatment period) and the whole number of rats was 32 .

a- Induction obesity period: It began from $1 \mathrm{st}-8^{\text {th }} \mathrm{wk}$; by feeding rats with HFD or HSD. The animals were divided into three groups; control, HFD and HSD group.

-Control group, maintained on standard normal rat chow diet along the all period of experiment (12 wk) (8 rats).

- HFD group was maintained on high fat diet (8 rats). HSD was giving high sucrose diet (16 rats).

b- Treatment period: It began form $8-12^{\text {th }}$ wk during this period each of HFD and HSD group was divided into two groups, (HFD) group continued on fat diet and $(\mathrm{HFD}+\mathrm{G})$ treated group maintained on high fat diet to 4 wks with administration of Garcinia treatment at dose (50 mg/rat/day) by stomach tube. While sucrose group divided into HSD maintained on high sucrose diet (8 rats) and (HSD+G) maintained on high sucrose diet with Garcinia treatment (8 rats).

Calculation of average food consumption per each rat was recorded daily by subtracting the amount of food remaining in each day from the measured amount of food provided at the previous day [6].

Calculation of energy intake of consumed diet: Metabolic energy of standard rat chow, HFD and HSD were 2813, 5130 and $3410 \mathrm{Kcal} / \mathrm{kg}$ respectively. The average energy intake of consumed diet was calculated by multiplying the average consumed diet by $2.813,5.130$ and 3.410 respectively according to consumed diet [6].

BMI for rats was measured every wk and calculated by dividing the body weight in gm by the length (nose to base of tail) in $\mathrm{cm}^{2}$. Also body weight was measured.

\section{2- Sampling and tissue preparation}

Blood samples were collected from medial canthus of the eye, via glass capillaries at fasting state. The samples were collected in dry glass centrifuge tubes, allowed to coagulate at room temperature and centrifuged at $3500 \mathrm{rpm}$ 
for 15 minutes at room temperature for separation of serum. The clear, non-haemolysed supernatant sera were separated using clean dry disposable plastic syringes and stored at $-20^{\circ} \mathrm{C}$ for subsequent biochemical measurements. Besides addition of sodium fluoride as anticoagulant for measuring of glucose in another blood sample.

Tissue sampling: At the end of experiment, rats were sacrificed by decapitation and abdominal incision was immediately done after taking of blood sample for separation of kidney and perirenal, mesentric and epididymal adipose tissues. Kidney was taken and washed by saline, dried by filter paper and weighed $0.5 \mathrm{gm}$ of this tissue then underwent homogenization for tissue biochemical analysis of catalase, MDA and NO.

\section{3- Biochemical assays of serum and tissue}

Plasma glucose was estimated according to the method of Trinder [7] using Stanbio Laboratory USA Kits. Serum urea [8] and creatinine levels [9] were measured colorimetrically using kits purchased from Diamond Diagnostic, Egypt. Serum was analyzed for lipid profile (TC, TG, LDL and HDL) levels and G6PD activity [10] by enzymatic colorimetric methods using assay kits (Bio-diagnostic Dokki, Giza, Egypt).

Renal and serum MDA levels were measured, as described by Mihara and Uchiyama [11]. Briefly, MDA was allowed to react with thiobarbituric acid that yielded red-coloured products, which were spectrophotometrically quantified by measuring the maximum absorption peaks at $532 \mathrm{~nm}$. Renal catalase (CAT) activity was determined according to the procedure of Cohen et al. [12]. Renal and/or serum NO levels were measured according to Miranda et al. [13].

\section{Statistical analysis}

Data were presented as mean \pm S.E.M and analyzed using one-way analysis of variance (ANOVA) followed by Tukey-Kramer methods for post-hoc analysis. A value of $\mathrm{p}<0.05$ was considered statistically significant. Correlation coefficient between the measured variables was tested where $(\mathrm{P})$ and $(\mathrm{r})$ values were recorded. Graph Pad Prism 5 software (San Diego, CA, USA) was used for statistical analysis.

\section{Results}

Effect of HFD, HSD and Garcinia on feed intake, BW and BMI In this study, feed intake (Figure 1 \& 2), BW (Figure 3) and BMI (Figure 4) were significantly increased during the period of the experiment in accompanied with significant increases in mesenteric, perirenal and epididymal adipose tissue in HFD and HSD compared with that of control group in rats. Garcinia treatment tended to decrease food intake, BW and BMI (Figure 2, 3, 4).

\section{Effect of HFD, HSD and Garcinia treatments on lipid} profile and kidney function

Serum urea and creatinine levels were significantly increased in HFD and HSD compared with those in control group. There were significant higher levels of urea and creatinine in HFD than HSD group $\mathrm{p} \leq 0.05$. In addition, TC, triglyceride and LDL levels were also significantly increased in group HFD and HSD compared with those in control group. Cotreatment of HFD and HSD-administered rats with Garcinia produce significant decrease in serum urea, creatinine, TC, triglyceride and LDL levels compared to their respective HFD and HSD-treated rats (Table 1 ).

\section{Effect of HFD, HSD and Garcinia treatments on plasma} glucose, G6PD and adipose tissues

Table (2) showed significant increase in glucose in HFD group compared with control and these levels were significantly lowered in HFD $+G$ than HFD group $\mathrm{p} \leq 0.05$. G6PD activities were significantly decreased in HFD while there were significant higher G6pD activities in HSD and HSD $+G$ groups $p \leq 0.05$ than control.

There were significant increases in mesenteric, perirenal and epididymal adipose tissues in HFD and HSD compared with that of control group in rats. Garcinia treatment tended to decrease adipose tissues weight compared with HFD and HSD group (Table 2).

The mesenteric, perirenal and epididymal fat-pad weights were reduced by $28 \%, 61 \%$ and $52 \%$ respectively in rats fed HFD $+\mathrm{G}$ than HFD rat $(\mathrm{p}<0.05)$. While the mesenteric, perirenal and epididymal fat-pad weights were reduced by $31 \%, 35 \%$ and $37 \%$ respectively in rats fed HSD+G than HSD rat $(\mathrm{p}<0.05)$ (Table 2).

\section{Effects of diet induced obesity \& treatment on oxidative stress (MDA) and catalase activity}

Feeding of HFD and HSD caused a significant increase in serum MDA level compared with control group. Treatment with Garcinia significantly decreased MDA level compared with those in group HFD and HSD. In renal tissues MDA level and catalase activity were significantly increased in HFD and HSD compared with control group and treatment with Garcinia ameliorated theses changes (Table 3).

\section{Effect of diet induced obesity \& treatment on serum and renal NO levels \\ HFD and HSD significantly lowered both serum and renal NO levels in comparison with control. Treatment with the Garcinia significantly elevated the No level of the rat consuming HFD and HSD (Table 3).}




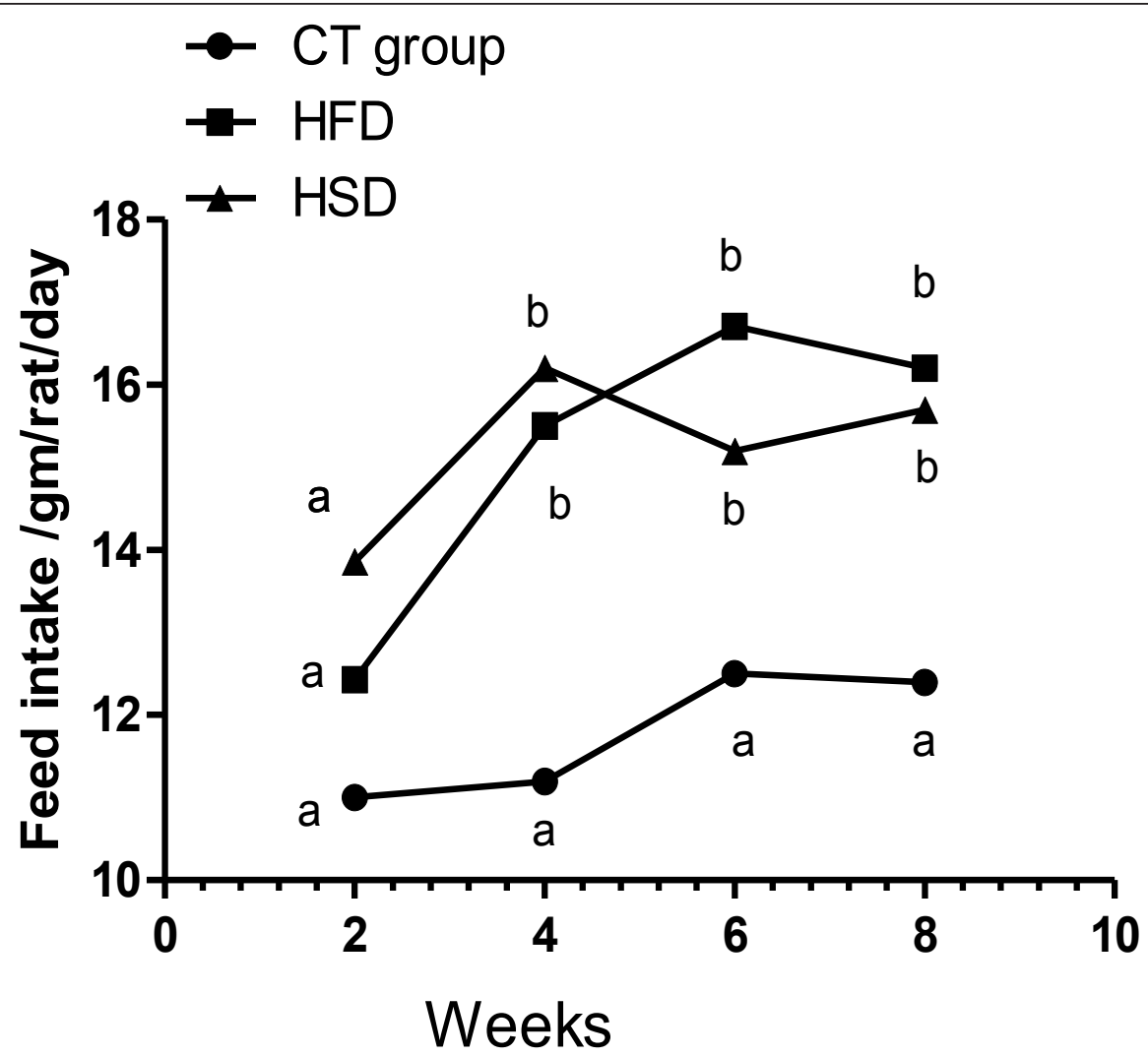

Figure 1 Means of feed intake (gm/day/rat) in the different groups of experiment $\left(0\right.$ to $\left.8^{\text {th }} \mathbf{w k}\right)$ in rats. Feed intake increased significantly in rats fed on HFD and HSD from 0 to $8^{\text {th }}$ wk compared with that of control group. Means have different letters indicate significant variation at $(P \leq 0.05)$, while the same letters indicate non significant variation.

\section{Correlation coeffecient between variables in different groups of rats}

There were significant and positive correlation between increased level of LDL and oxidative stress which was demonstrated by high level of MDA $(P=0.02 \& r=0.94)$ (Table 4). Also were significant positive correlations between TG and Oxidative renal MDA marker $(\mathrm{P}=0.01$ $\& \mathrm{r}=0.96)$ (Table 4).

The current data showed negative correlation between renal NO with renal MDA $(\mathrm{P}=0.046$ and $\mathrm{r}=-0.88)$ and renal catalase $(P=0.005$ and $r=-0.97)$ as indicator for oxidative stress in kidney. Moreover there were significant positive correlations among BMI, kidney functions as creatinine, $(P=0.98 \& r=0.002)$, urea $(P=0.006 \& r=0.97)$.

\section{Discussion}

Effect of diet induced obesity and treatment on feed intake, body weight and BMI

In this study there was significant higher food intake in both free diet of HFD and HSD during $4^{\text {th }}, 6^{\text {th }}$ and $8^{\text {th }}$ wk (Figure 1) and $10^{\text {th }} \& 12^{\text {th }}$ wk (Figure 2) compared to control diet and this is may be due to HFD causing hyperphagia which similar to human cafeteria diet [14].
The mechanisms for how saturated fat and sugar-based beverages contribute to human obesity are clearly in rats on an HF choice diet, plasma leptin concentrations and proopiomelanocortin mRNA increased and neuropeptide Y mRNA decreased [15].

Dietary fat possesses a number of characteristics that may contribute to its overconsumption. Both palatability and energy density were contribute to fat hyperphagia and reduced satiation signaling accompanying HFD consumption which could contribute to overconsumption and often leads to obesity. Although HF adaptation promotes short-term overconsumption of a high-energy food (i.e., reduced satiation), it also appears to offer a vital influence in the control of energy metabolism. Long-term HFD eating may promote excessive shortterm energy intake by reducing sensitivity to the satiating properties and thus consume more food [16].

Rats fed high-saturated fat had both hyperglycaemia and hyper-triacylglycerolemia, the same as our model while rats fed high n-3 PUFA only had hyperglycaemia. In this concern various forms of dietary fat differentially change the expression of neuropeptide genes involved in energy homeostasis [17]. 


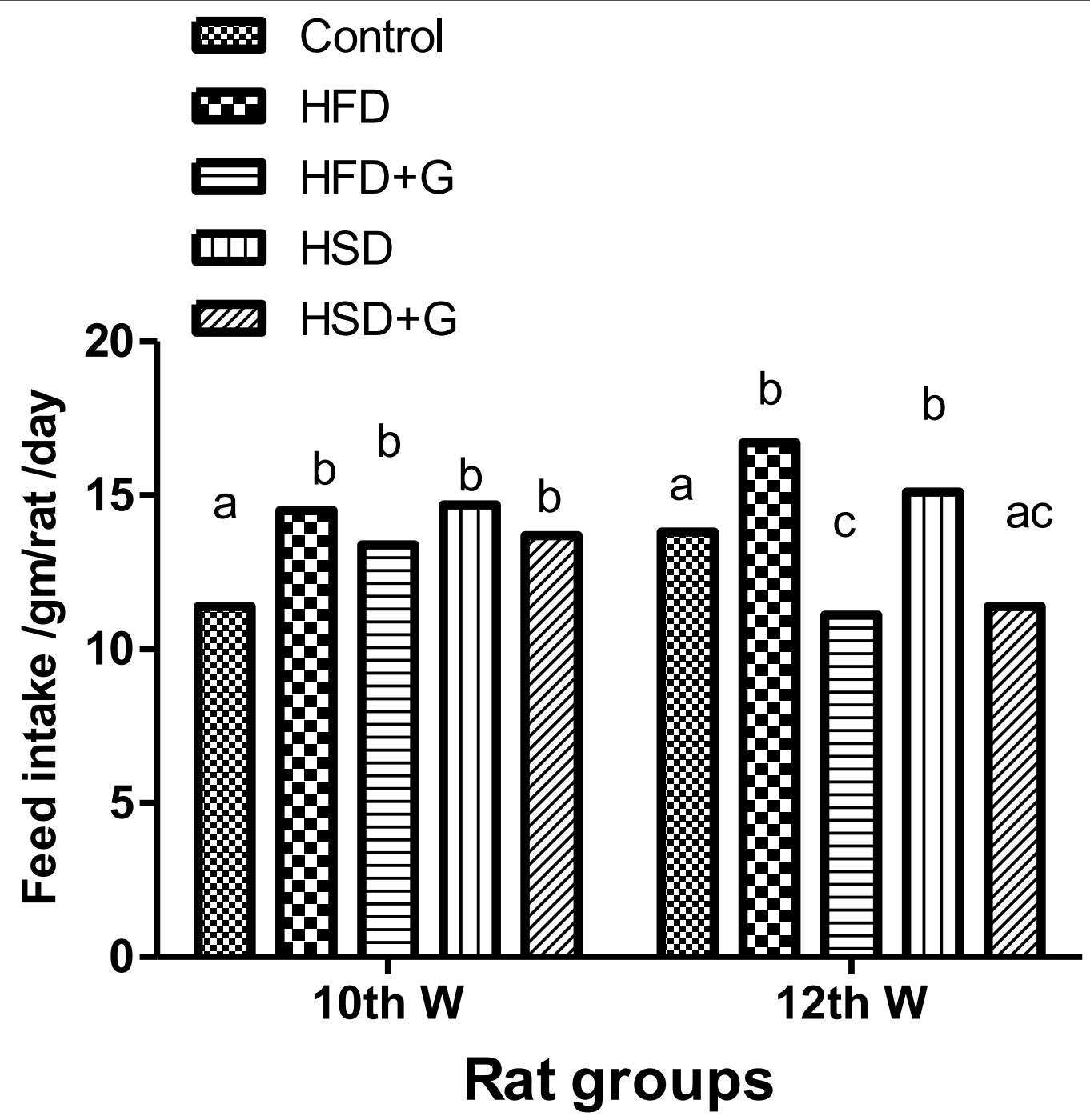

Figure 2 Means of feed intake (gm/day/rat) in different groups of experiment $\left(8^{\text {th }}-12^{\text {th }} \mathbf{w k}\right)$ in rats. Feed intake increased significantly in rats fed on HFD and HSD from $8^{\text {th }}-12^{\text {th }}$ wk compared with control group. Garcinia treatment tended to decrease feed intake compared with HFD and HSD group. Means have different letters indicate significant variation at ( $P \leq 0.05)$.

Higher feed intake in HSD may be due to hyperphagia induced by sucrose which affect appetite centers in hypothalamic neuropeptids Y, (NPY) and proopinne melanocortin (POMC) both receive information about nutritional status and level of energy storage through insulin and leptin signaling mediated by specific receptor located POMC and NPY neurons. Sucrose consumption increase calories intake through up regulation of hypothalamic CB1 mRNA and down regulation of NPY mRNA [17]. Also consumption of sucrose solution results in high body weight gain through activation of hunger signals and depression of satiety signals which support our results.

The current data showed significant increase in body weight and BMI especially in the $8^{\text {th }}$ wk in HFD and
HSD (Figure 3 \& 4). Giving of HFD through canula showed significant increase in body weight in accordance with Akiyama et al. [18].

Increased BW and BMI may be due to increase caloric intake resulting in more adipose tissue deposition than starch diet this result varies between researches due to sex, age, strain. Furthermore HSD appear to induce mitochondrial dysfunction in adipose tissue, which may be related to greater weight gain and metabolic impairment $[19,20]$.

Garcinia ameliorated feed intake (Figure 2) and significantly decreased BW and BMI (Figure $3 \& 4$ ). These effects of HCA in Garcinia achieved by increasing release/availability of 5 hydroxy tryptamine, or serotonin, also enhance serotonin uptake in the brain [5]. 


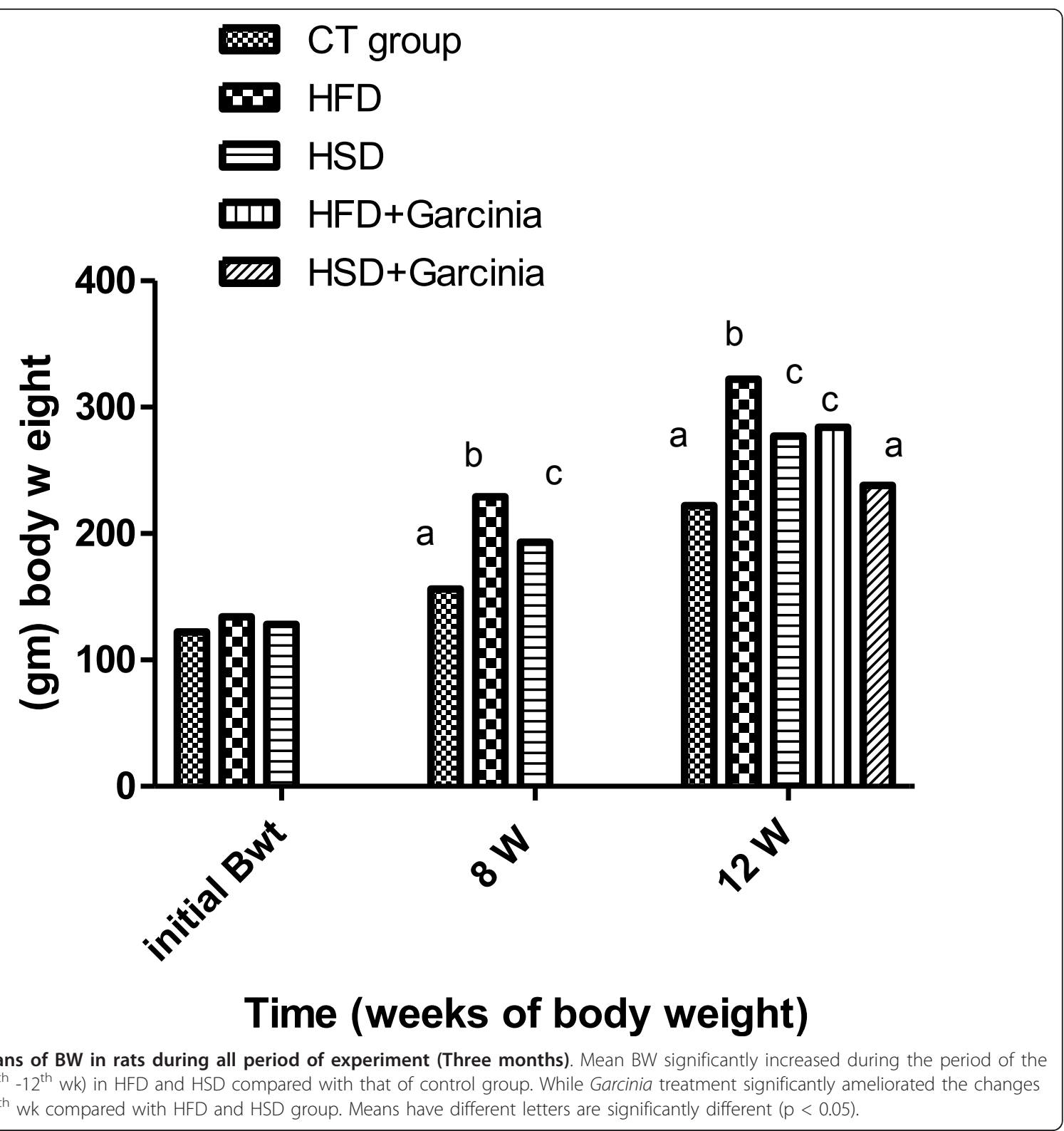

Serotonin, a neurotransmitter implicated in the regulation of eating behavior, appetite control and weight management by curbing appetite, reduction of food intake and inhibiting body fat biosynthesis [21].

\section{Effect of diet induced obesity and treatment on lipid profiles}

HFD and HSD significantly increased TC, TG and LDL levels in comparison with control group (Table 1). These findings may be owed to high fat from beef tallow induced hypercholesterolemia [22]. Dietary sucrose significantly produced hypertriglyceridemia across rats' life span that had free food access or were calorie restricted and may be due to increased secretion of TG and decreased its catabolism [23]. HSD induces hepatic synthesis of TG from glucose and transport it to blood through VLDL and stored in adipose tissue.

There were significant and positive correlation between increased level of LDL and oxidative stress which was demonstrated by high level of MDA ( $\mathrm{P}=$ $0.02 \mathrm{r}=0.94$ ) (Table 4) and high MDA from lipid peroxidation causing oxidative stress which generally result in increase oxidation of LDL.

Garcinia treatment produces decrement in LDL, TG and TC serum levels. These findings occur as a result of inhibiting effect of hydroxy citric acid on ATP citrate lyase, an enzyme which catalyses extramitochondrial cleavage of citrate to oxaloacetate and acetyl COA. The 


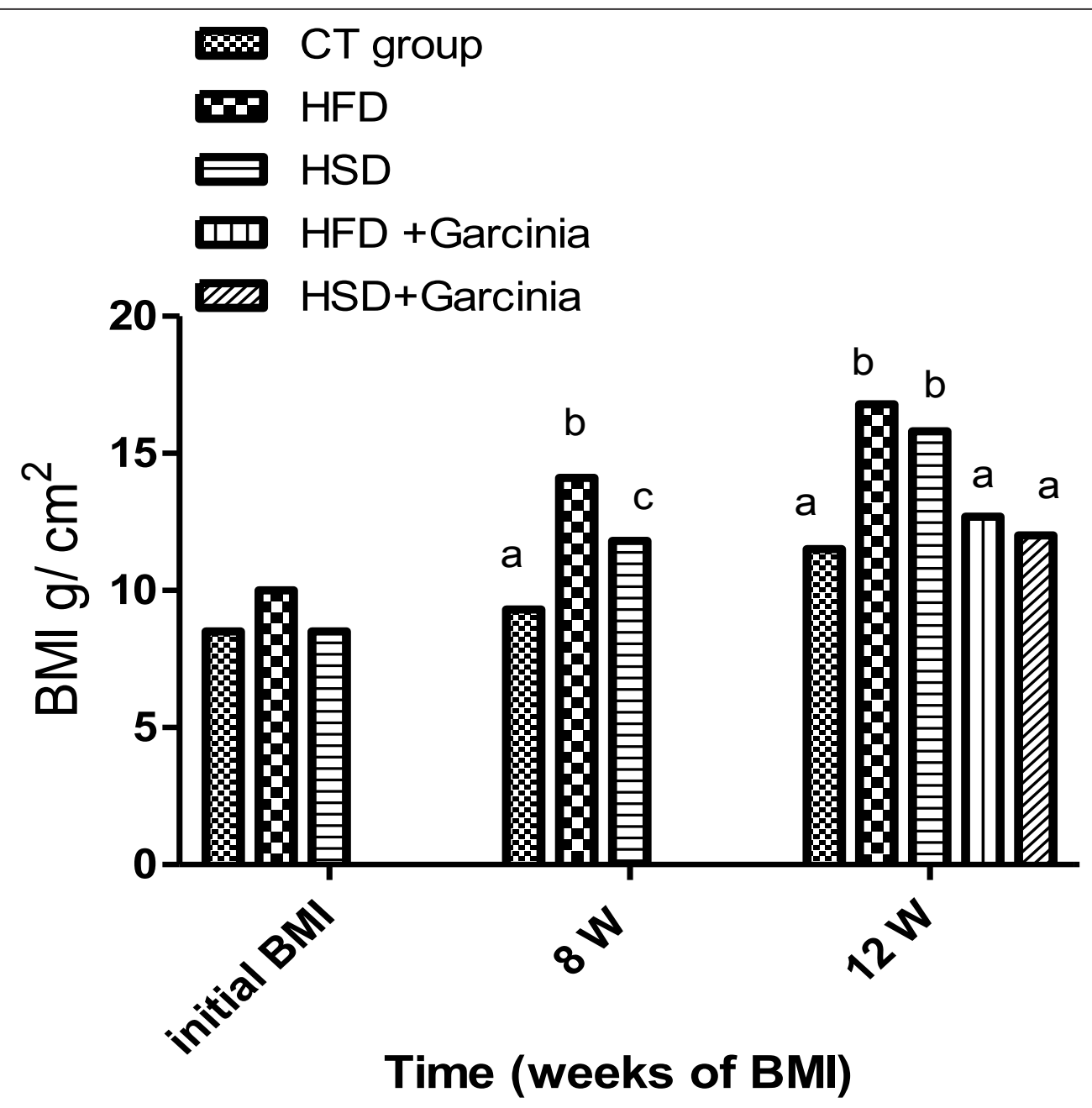

Figure 4 Means of BMI in rats during the period of the experiment (Three months). BMI significantly increased during the period of the experiment in HFD and HSD compared with that of control group in rats. Administration of Garcinia significantly improved BMI compared with HFD and HSD group. Means not sharing common letter are significantly different $(p<0.05)$.

Table 1 Effect of HFD, HSD and Garcinia treatments on kidney function and lipid profile in different groups of rats

\begin{tabular}{llllll}
\hline & Control & HFD & HFD + G & HSD & HSD+ G \\
\hline Creatinine (mg/dl) & $0.3 \pm 0.02^{\mathrm{a}}$ & $0.8 \pm 0.03^{\mathrm{b}}$ & $0.4 \pm 0.04^{\mathrm{ac}}$ & $0.8 \pm 0.04^{\mathrm{b}}$ & $0.3 \pm 0.04^{\mathrm{ac}}$ \\
\hline Urea $(\mathbf{m g} / \mathbf{d l})$ & $19 \pm 1.5^{\mathrm{a}}$ & $38 \pm 2.4^{\mathrm{b}}$ & $14 \pm 0.91^{\mathrm{ac}}$ & $30 \pm 3.9^{\mathrm{d}}$ & $15 \pm 1.8^{\mathrm{ac}}$ \\
\hline TC $(\mathbf{m g} / \mathbf{d l})$ & $81 \pm 1.4^{\mathrm{a}}$ & $351.01 \pm 4.6^{\mathrm{b}}$ & $112 \pm 1.3^{\mathrm{c}}$ & $320 \pm 3.3^{\mathrm{b}}$ & $172 \pm 4.5^{\mathrm{e}}$ \\
\hline TG $(\mathbf{m g} / \mathbf{d l})$ & $85 \pm 2.3^{\mathrm{a}}$ & $384.01 \pm 11^{\mathrm{b}}$ & $203 \pm 2.7^{\mathrm{c}}$ & $350 \pm 3.9^{\mathrm{bd}}$ & $201 \pm 10^{\mathrm{ce}}$ \\
\hline LDL $(\mathbf{m g} / \mathbf{d l})$ & $26 \pm 2.1^{\mathrm{a}}$ & $284 \pm 5.5^{\mathrm{b}}$ & $61 \pm 3^{\mathrm{c}}$ & $212 \pm 4.9^{\mathrm{d}}$ & $61 \pm 3.9^{\mathrm{c}}$ \\
\hline HDL $(\mathbf{m g} / \mathbf{d l})$ & $44 \pm 1.8^{\mathrm{a}}$ & $73 \pm 2.2^{\mathrm{a}}$ & $72 \pm 2.8^{\mathrm{a}}$ & $77 \pm 6.5^{\mathrm{a}}$ & $66 \pm 2.3^{\mathrm{a}}$ \\
\hline
\end{tabular}

Each value is the mean \pm SEM. Means have different superscript letters indicate significant variation at $(P \leq 0.05)$, while the same letters indicate non significant variation.

Serum urea and creatinine levels were significantly increased in HFD and HSD compared with control group. In addition, TC, triglyceride and LDL levels were also significantly increased in group HFD and HSD compared with those in control group. HFD and HSD-administered rats with Garcinia produced significant decrease in serum urea, creatinine, TC, triglyceride and LDL levels compared to their respective HFD and HSD-treated rats.

Effect of HFD, HSD and Garcinia treatments on blood glucose, G6pD and adipose tissues during three months in different groups of rats. 
Table 2 Effect of HFD, HSD and Garcinia treatments on blood glucose, G6pD and adipose tissues during three months in different groups of rats

\begin{tabular}{|c|c|c|c|c|c|}
\hline & Control & HFD & HFD+G & HSD & $\mathrm{HSD}+\mathrm{G}$ \\
\hline Glucose (mg/dl) & $59.0 \pm 1.6^{a}$ & $111.0 \pm 4.1^{b}$ & $91.0 \pm 3.9^{c}$ & $71.0 \pm 3.2^{\mathrm{ad}}$ & $63.0 \pm 3.6^{\mathrm{ad}}$ \\
\hline G6pD (mU/ml) & $0.25 \pm 0.02^{a}$ & $0.14 \pm 0.01^{b}$ & $0.12 \pm 0.002^{b}$ & $0.53 \pm 0.04^{c}$ & $0.54 \pm 0.03^{c}$ \\
\hline Mesenteric fat (g) & $1.7 \pm 0.19^{a}$ & $3.6 \pm 0.41^{b}$ & $2.6 \pm 0.29^{c}$ & $3.2 \pm 0.29^{c}$ & $2.2 \pm 0.33^{e}$ \\
\hline Perirenal fat (g) & $2.4 \pm 0.29^{a}$ & $3.3 \pm 0.23^{b}$ & $1.3 \pm 0.31^{\mathrm{ac}}$ & $3.4 \pm 0.2^{b c}$ & $2.2 \pm 0.3^{d}$ \\
\hline Epididymal fat (g) & $0.88 \pm 0.06^{a}$ & $2.3 \pm 0.075^{b}$ & $1.1 \pm 0.041^{c}$ & $1.9 \pm 0.025^{b}$ & $1.2 \pm 0.1^{d c}$ \\
\hline
\end{tabular}

Each value is the mean \pm SEM. Means have different superscript letters indicate significant variation at $(P \leq 0.05)$, while the same letters indicate non significant variation.

Plasma glucose was significant increased in HFD group compared with control and these levels were significantly lowered in HFD+G than HFD group $p \leq 0.05$. There were significant increases in G6pD activities in HSD and HSD+G groups p $\leq 0.05$ than control, while giving Garcinia ameliorated these changes. Mesenteric, perirenal and epididymal adipose tissue weight were significantly increased in HFD and HSD compared with control group.

The mesenteric, perirenal and epididymal fat-pad weights were reduced by $28 \%, 61 \%$ and $52 \%$ respectively in rats fed HFD+G than HFD rat ( $p<0.05$ ). While the mesenteric, perirenal and epididymal fat-pad weights were reduced by $31 \%, 35 \%$ and $37 \%$ respectively, in rats fed HSD+G than HSD rat. Garcinia treatment tended to decrease adipose tissues weight compared with HFD and HSD.

Effect of HFD, HSD and Garcinia treatments on renal catalase and both serum and renal MDA and NO levels in rats.

produced acetyl COA is used in fatty acid synthesis, TC and TG synthesis. The current data suggested that Garcinia has hypolipidemic action causing significant hypocholesterolemic and hypotriglycerdemic effect.

\section{Effect of HFD, HSD and treatment on kidney function}

Lifestyle factors and diet play a role in the development of kidney disease in several stages including, progress of obesity and the metabolic syndrome and occurrence of obesity-related glomerulopathy.

HFD and HSD caused significant elevation in serum creatinine a specific indicator of glomerular function and urea level (Table 1) owing to consumption of HFD and HSD which result in metabolic syndrome marked by obesity, hyperlipidemia and associated with oxidative stress and nitric oxide inactivation by reactive oxygen species (ROS) and diminish NO bioavailability [24] which leading to renal dysfunction, characterizing by high level of creatinine and blood urea nitrogen [25].

Using HFD (32\%) for $10 \mathrm{wk}$, about one-half develop obesity and mild hypertension, oxidative stress and impaired renal function. Also hydroxynonenal protein adducts in the kidney were highly increased, indicating oxidative stress in the renal tissue [26].

In obese subject, increased serum creatinine was to be observed suggesting that obesity caused elevation in renal function test and produce proteinuria, concomitantly with other risk factors such as hypertension, diabetes and dyslipidemia [27].

Obesity is recently acknowledged as an important independent risk factor for kidney disease. This risk is probably explained by renal intracellular lipid accumulation [28]. Also metabolic disorders in obesity associated with high blood pressure, poor glycemic control, dyslipidemia and smoking were considering risk factor for susceptibility of chronic renal disease (CRD). Glomerular hypertension and endothelial dysfunction were regarded as common mechanism of CRD rennin-angiotensin system.

Renal triglyceride accumulated in obese rats accompanied by hypoalbuminemia and elevated blood urea nitrogen. Dysregulated gene expression may result in increased

Table 3 Effect of HFD, HSD and Garcinia treatments on renal catalase and both serum and renal MDA and NO levels in rats

\begin{tabular}{|c|c|c|c|c|c|}
\hline Parameters & Control & HFD & HFD+G & HSD & $\mathrm{HSD}+\mathrm{G}$ \\
\hline Renal Catalase (U/gm) & $4.4 \pm 0.29^{a}$ & $14.2 \pm 1.3^{b}$ & $8.3 \pm 0.64^{c}$ & $14.5 \pm 0.34^{b}$ & $9.8 \pm 0.67^{c}$ \\
\hline S. MDA (nmol/ml) & $2.6 \pm 0.22^{a}$ & $4.9 \pm 0.31^{b}$ & $2.4 \pm 0.42^{a}$ & $7.3 \pm 0.37^{c}$ & $4.1 \pm 0.44^{b}$ \\
\hline Renal MDA (nmol/g) & $1.2 \pm 0.15^{a}$ & $6.6 \pm 0.27^{b}$ & $3.7 \pm 0.41^{\mathrm{cd}}$ & 4.8. $\pm 0.5^{c}$ & $2.7 \pm 0.30^{\mathrm{ad}}$ \\
\hline S. NO ( $\mu \mathrm{mol} / \mathrm{L})$ & $2.8 \pm 0.28^{a}$ & $2.2 \pm 0.14^{b}$ & $3.7 \pm 0.24^{\mathrm{ac}}$ & $1.6 \pm 0.26^{d}$ & $6.8 \pm 0.53^{e}$ \\
\hline Renal NO ( $\mu \mathrm{mol} / \mathrm{g})$ & $1.3 \pm 0.09^{a}$ & $0.7 \pm 0.04^{b}$ & $0.9 \pm 0.025^{c}$ & $0.6 \pm 0.03^{b}$ & $0.8 \pm 0.02^{c}$ \\
\hline
\end{tabular}

Each value is the mean \pm SEM. Means have different superscript letters indicate significant variation at $(P \leq 0.05)$, while the same letters indicate non significant variation.

Feeding of HFD and HSD caused a significant increase in serum MDA level compared with control group. Treatment with Garcinia significantly decreased MDA level compared with those in group HFD and HSD. In renal tissues MDA level and catalase activity were significantly increased in HFD and HSD compared with control group and treatment with Garcinia ameliorated theses changes.

Both serum and renal NO levels significantly lowered in HFD and HSD in comparison with control. Treatment with the Garcinia significantly elevated the No level in rats consuming HFD and HSD. 
Table 4 Correlation coeffecient between variables in different groups of rats

\begin{tabular}{|c|c|c|c|c|c|c|c|}
\hline & TG & LDL & Creatinine & BMI & BW & Renal Catalase & Renal NO \\
\hline TG & - & $\begin{array}{l}P=0.01 \\
r=0.95\end{array}$ & $\begin{array}{l}P=0.02 \\
r=0.93\end{array}$ & $\begin{array}{l}P=0.01 \\
r=0.96\end{array}$ & $\begin{array}{l}P=0.03 \\
r=0.92\end{array}$ & $\begin{array}{l}P=0.003 \\
r=0.98\end{array}$ & $\begin{array}{l}P=0.02 \\
r=-0.94\end{array}$ \\
\hline Creatinine & $\begin{array}{l}P=0.02 \\
r=0.93\end{array}$ & $\begin{array}{l}P=0.01 \\
r=0.96\end{array}$ & - & $\begin{array}{l}P=0.002 \\
r=0.98\end{array}$ & $\begin{array}{l}P=0.04 \\
r=0.89\end{array}$ & $\begin{array}{l}P=0.04 \\
r=0.89\end{array}$ & NS \\
\hline Urea & $\begin{array}{l}P=0.04 \\
r=0.88\end{array}$ & $\begin{array}{l}P=0.002 \\
r=0.98\end{array}$ & $\begin{array}{l}P=0.02 \\
r=0.94\end{array}$ & $\begin{array}{l}P=0.006 \\
r=0.97\end{array}$ & NS & NS & NS \\
\hline Renal MDA & $\begin{array}{l}P=0.01 \\
r=0.96\end{array}$ & $\begin{array}{l}P=0.02 \\
r=0.94\end{array}$ & $\begin{array}{l}P=0.042 \\
r=0.89\end{array}$ & $\begin{array}{l}P=0.02 \\
r=0.94\end{array}$ & $\begin{array}{l}P=0.01 \\
r=0.97\end{array}$ & $\begin{array}{l}P=0.04 \\
r=0.90\end{array}$ & $\begin{array}{l}P=0.046 \\
r=-0.88\end{array}$ \\
\hline Renal Catalase & $\begin{array}{l}P=0.003 \\
r=0.98\end{array}$ & $\begin{array}{l}P=0.03 \\
r=0.90\end{array}$ & $\begin{array}{l}P=0.04 \\
r=0.89\end{array}$ & $\begin{array}{l}P=0.03 \\
r=0.91\end{array}$ & NS & - & $\begin{array}{l}P=0.005 \\
r=-0.97\end{array}$ \\
\hline Renal NO & $\begin{array}{l}P=0.04 \\
r=-0.90\end{array}$ & NS & NS & NS & NS & $\begin{array}{l}P=0.005 \\
r=-0.97\end{array}$ & - \\
\hline BMI & $\begin{array}{l}P=0.01 \\
r=0.96\end{array}$ & $\begin{array}{l}P=0.001 \\
r=0.9\end{array}$ & $\begin{array}{l}P=0.002 \\
r=0.98\end{array}$ & - & $\begin{array}{l}P=0.03 \\
r=0.92\end{array}$ & $\begin{array}{l}P=0.03 \\
r=0.91\end{array}$ & NS \\
\hline
\end{tabular}

$r=$ Value of correlation coefficient $P \leq 0.05$ indicate significant variation.

There were significant and positive correlation between increased level of LDL and oxidative stress which was demonstrated by high level of MDA ( $P=0.02$ \& $r=0.94)$ (Table 4). Also were significant positive correlations between TG and Oxidative renal MDA marker $(P=0.01 \& r=0.96)($ Table 4$)$.

The current data showed negative correlation between renal NO with renal MDA $(P=0.046$ and $r=-0.88)$ and renal catalase $(P=0.005$ and $r=-0.97)$ as indicator for oxidative stress in kidney. Moreover there were significant positive correlations among BMl, kidney functions as creatinine $(\mathrm{P}=0.98 \& \mathrm{r}=0.002)$ and urea $(P=0.006 \& r=0.97)$.

renal collagen cross-linking and lipid accumulation, that may be associated with development of nephropathy in the animal model of type 2 diabetes and obesity [29].

Feeding HFD to rat resulted in macroscopical and microscopical changes in kidney weight, total kidney volume, changes in medulla and cortex, glomerulus, proximal and distal convoluted tubules [30].

Visceral and renal fat accumulation through consumption of a HFD leads to marked renal sympathetic activation, which is related to increased responsiveness to central sympathoexcitatory effects of leptin that contributes to the development of hypertension [31].

Insulin resistance and increased sympathetic nerve activity were implicated in development of glomerular hypertension and endothelial dysfunction [32]. Furthermore hyperinsuliemia induced by obesity cause relaxation of afferent arterioles leading to hyperfiltration of gloumerulus and renal damage [33], which explained the defect in renal functions in our model of HFD and HSD.

Garcinia treatment enhances renal function as a result of HCA-SX derived from Garcinia cambogia (HCA-SX, Super CitriMax) which attenuated the increased oxidative stress biomarker through reducing lipid peroxidation (MDA) and declining lipid profiles and level of oxidized LDL which generally improved kidney function [34].

\section{Effect of diet induced obesity and treatment on blood glucose level}

Obesity and type 2 diabetes mellitus is correlated with each other, in our experiment significant hyperglycaemia occurred due to HFD (Table 2), which resulted from free fatty acid release from adipose tissue causing peripheral and hepatic insulin resistance initiating T2DM. Furthermore, FFA impair muscle uptake of glucose by competitive inhibition [[18] \&[35]].

HSD has no significant effect on blood glucose level, that result was in accordance with Fukuchis et al. [35] who reported that rats fed a sucrose diet for 4 wks had significantly larger visceral fat pads and hypertriglyceridemia, however, neither plasma glucose nor insulin levels were significantly higher, while hyperglycemia and insulin resistance occur after 20 wks of feeding HSD.

Relationship between renal disorder and obesity in HFD group was confirmed by hyperglycemia, insulin resistance, decreased production of renal NO and increased oxidative stress which implicated in progression of diabetic nephropathy [36].

Garcinia significantly improved the hyperglycemia, in HFD, by declining insulin resistance [37], in this concern Garcinia could be used for management of diabetes, by increasing metabolic pathway via rising glucose oxidation through improving insulin action; also Garcinia promotes glycogenesis and lipid oxidation.

\section{Effect of diet induced obesity and treatment on G6PD activity}

G6PD activities were significantly decreased in HFD (Table 2). These findings may be due to hyperglycemia with HFD caused activation of protein kinase A and subsequent increased phosphorylation and inhibition of G6PD activity and hence decreased NADPH which therefore led to increased oxidative stress [38]. G6PD activity and accordingly NADPH level was significantly decreased in diabetic nephropathy. 
Our data show that hyperglycaemia during HFD can reduce G6PD activity. Conversely, G6PD insufficiency can promote oxidative stress and impairment of insulin secretion by beta cells. It is suggested that reduced G6PD activity and HFD can aggravate each other, and HFD could be aetiologically associated with reduced G6PD activity.

Lipid peroxidation was significantly increased, while G6PD activity was decreased in HFD group. Oxidative stress due to increased oxidant production and/or decreased antioxidant activity is a critical underlying mechanism. The principal intracellular reductant is NADPH whose production is mainly dependent on G6PD activity.

There was significant increase in G6pD activities in HSD and HSD+G groups $\mathrm{p} \leq 0.05$ compared with control (Table 2) which may be attributed to metabolic alteration produced from sucrose rich diet, among these changes increased level of G6PD which is the first enzyme of hexose monophsphate pathway and induction of G6pD in paranchymal cells that support the escalating needs for NADPH for synthesis of fatty acids [39].

\section{Effect of diet induced obesity and treatment on adipose tissue}

Our data indicated significant increases in mesenteric, perirenal and epididymal adipose tissues in HFD and HSD group (Table 2). HFD increases the weight of different fat depots resulting in significant decrease in OB-Rb mRNA level [40].

Adipose tissue functions as an endocrine organ, secreting hormones/cytokines (e.g., leptin) which may trigger renal sodium retention by activating the reninangiotensin. Furthermore, excess perirenal and visceral adipose tissue may physically compress the kidneys, increasing intrarenal pressures and tubular reabsorption. Ultimately, continuous obesity causes renal structural alteration, glomerular hypertrophy and occasionally focal segmental glomerulosclerosis [41].

$\mathrm{HCA}$, an active ingredient of Garcinia, reduces food consumption probably by diverting fatty acids and carbohydrates that would have become fat in the liver into hepatic glycogen [5]. This metabolic alteration may send a signal to the brain that result in rising serotonin level concomitant with a reduced appetite. Moreover, using Garcinia causes dispersion of fat which facilitate action of lipase on adipose tissue [42], suppresses body fat accumulation [43], inhibit cytoplasmic lipid accumulation and regulates adipogenesis, so eliminates body fat.

\section{Effect of diet \& treatment on renal catalase activity}

Renal catalase activity was significantly increased in HFD and HSD (Table 3). These findings occur in response to oxidative stress and important to balance the elevated ROS resulting from the activation of biochemical pathway leading to increased level of ROS and increased lipid peroxidation, producing oxidative stress [35]. Increased catalase activity was indicative of elevated ROS and consequently higher oxidative stress.

The increment in renal catalase and MDA offer better understanding and evidence for the relation between obesity and oxidative stress where increased ROS levels generally stimulate antioxidant system as a compensated defense mechanism and are an important trigger for insulin resistance. Some researchers reported that when exposure of alpha cells of pancreas to diabetogenic substance there was increase of catalase activety [44].

Renal catalase activity reduced after using Garcinia, suggesting the role of HCA-SX to attenuate the increase in oxidative stress, via decreasing feed intake, indicating the antioxidant effect of Garcinia.

\section{Effect of diet induced obesity and treatment on serum and renal MDA}

HFD and HSD generated significant increase in blood and renal levels of MDA compared to control group, while Garcinia, significantly decreased levels of serum MDA and renal MDA compared with HFD and HSD group(Table 3) as result of HCA-SX that inhibit ATP citrate lyase which catalyse extramitochondrial cleavage of citrate to oxaloacetate giving acetyl COA which used in fatty acid synthesis, suggesting that Garcinia has hypolipidemic action and reduces MDA in kidney, so HCA improves lipid peroxidation [34].

Feeding HFD and HSD raised LDL, TC, TG and renal lipid peroxidation (MDA) level which affect on the kidney because MDA act as tissue toxicant metabolites. These changes were monitored by increased level of urea and creatinine, whereas using Garcinia as antiobesity agent decreased TC, LDL and improved kidney function.

A number of reports suggested that ROS overproduction in the kidneys [36], heart and arteries [45], was involved in obesity-induced hypertension. However, the role of ROS and NO in the kidney has not been clarified.

The current data showed that TG positively correlated significantly with renal MDA $(\mathrm{P}=0.01$ and $\mathrm{r}=0.96)$ and catalase $(\mathrm{P}=0.003$ and $\mathrm{r}=0.98)$ as indicators of oxidative stress, while negatively correlated with renal $\mathrm{NO},(\mathrm{P}=0.04$ and $\mathrm{r}=-0.90)$ (Table 4$)$ suggesting an association between renal lipid peroxidation and obesity associated nephropathy. Furthermore, oxidative stress was increased in HFD and HSD groups as MDA level and catalase activity were increased, while NO level was decreased. 


\section{Effect of diet induced obesity \& treatment on serum and renal NO level}

HFD and HSD significantly decreased serum and renal NO compared with control group (Table 3). Obesity associated with hyperglycemia is a key factor that contributes to the development of diabetes-related microvascular disease both cyclooxygenase 1 and cyclooxygenase 2 and play a key role in the regulation of cardiovascular function, this increment resulted in rise of oxidative stress and reduction in NO generation in microvessels endothelial cells [46], suggesting that NO deficiency may contribute to renal vascular congestion and the renal dysfunction progression.

NO synthetase activity in the HFD group was decreased associated with diminished L-arginine transportation [47]. Also adipose tissue has a role in secreting factor that impairs endothelial dependent dilatation via inhibition of NO synthase mediating NO production [48]. Consequently, Garcinia that decreased TG results in replenish of NO level to normal.

NO signalling, which is involved in the regulation of food intake and insulin signalling, is altered in obesity and diabetes. It was suggested that hyperglycaemia impairs glucose and insulin regulation of $\mathrm{NO}$ production which occur through AMP-activated protein kinase [49].

Our data showed negative correlation between renal NO with renal MDA $(P=0.046$ and $r=-0.88)$ and renal catalase $(\mathrm{P}=0.005$ and $\mathrm{r}=-0.97)$ as indicator for oxidative stress in kidney tissues (Table 4). These are considering novel data of our known, suggesting that renal oxidative stress associated with lower renal NO initiating glomerular vasoconstriction and favour nephropathy.

We found that NO negatively correlated with MDA and hence its inactivation by ROS and functional NO deficiency in our model. So enhanced ROS-mediated inactivation and sequestration of $\mathrm{NO}$ which may contribute to the reduction of bioactive $\mathrm{NO}$ in obesity and hypertriglyceridemia.

NO plays an important role in the regulation of renal blood flow to the renal medulla and in the tubular regulation of sodium excretion. Rats fed HFD, resulted in decrease in serum and renal NO production (Table 3), indicating that obese rats are more liable to develop hypertension. Furthermore, HFD -induced defects in NO production may promote the salt-sensitivity of blood pressure, which appears to require more NO to maintain blood pressure during a salt challenge [50].

NO decreased in HFD and HSD, initiate vasoconstriction that affect the kidney and using methods for controlling obesity generate increased levels of NO and improving state of the involved organs that apparent in our results due to action of $\mathrm{NO}$ as vasodilator via using Garcinia.
Physical activity as a method of management of obese person prevent cardiovascular disease by increasing $\mathrm{NO}$ production and lessening NO inactivation [51], which could be comparable with our model of Garcinia used as antiobesity drugs.

Our rat's model was developed obesity and hyperglycemia by 12 wks on HFD and HSD diet. They also developed hypercholesterolemia, profound hypertriglyceridemia, high urea and creatinine, and renal function disorders. Furthermore, serum NO production was decreased, and homogenates from kidney demonstrated reduced NO. Our data offer novel insights into potential mechanisms of renal dysfunction, oxidative stress and NO in obesity and demonstrate the efficacy of HCA-SX in renal protection and weight management.

Collectively, feeding HFD and HSD to rats resulted in significant elevating in BW, BMI, feed intake, adipose tissue, blood levels of glucose, TG, TC, LDL, MDA and catalase activity while significantly, decreased serum and renal NO levels in our model. All of these parameters implicated in renal disorders, consequently produce adverse effect on kidney, while administration of Garcinia improves these changes as, HCA-SX attenuates the increase in oxidative stress so improves lipid peroxidation which is cytotoxic by reducing free-radical production and by increasing nitric oxide production/availability.

\section{Conclusions}

We could concluded that HFD and HSD induced obesity, represent the best available rat model to study nephropathy, which riskily affect the kidney via elevating urea, creatinine, TG, LDL and glucose and that the renal injuries were associated with increased oxidative stress monitored by increased MDA level and catalase activity whereas NO production decreases while using garcinia cambogia improved the levels of blood lipid profile, oxidative stress, biomarkers and glucose so improving kidney function. This study provides novel in vivo evidences of the protective effects of Garcinia against obesity-induced nephropathy.

\section{Acknowledgements}

This study was supported by fund from Beni Suef University, Beni Suef, Egypt. We appreciate the help and advice of Prof. Dr. Omaima A Ragab for kind cooperation.

\section{Author details}

'Biochemistry Department, Faculty of Vete. Medicine, Beni-Suef University, Beni-Suef, Egypt. ${ }^{2}$ Clinical pathology Department, Faculty of Vete. Medicine, Beni-Suef University, Beni-Suef, Egypt.

\section{Authors' contributions}

KA, HK and MA carried out experimental work; biochemical analysis, statistical analysis, interpretation and discussion of the results related to their part of the work. KA conceived, design and planning of the study, wrote the 
paper, drafting and revision of the manuscript. All authors read and approved the final manuscript.

\section{Competing interests}

The authors declare that they have no competing interests.

Received: 12 December 2010 Accepted: 14 January 2011

Published: 14 January 2011

\section{References}

1. Galal OM: The nutrition transition in Egypt: obesity, under nutrition and the food consumption context. Public Health Nutr 2002, 5:141-148.

2. Kim MS, Kim Jk, Kwon DY, Park R: Antiadipogenic effect of garcinia extract on lipid droplets accumulation and the expression of transcription factor. Bio factor 2004, 22:193-196.

3. Amin KA, Nagy MA: Effect of Carnitine and herbal mixture extract on obesity induced by high fat diet in rats. Diabetol Metab Syndr 2009, 16,1(1):17.

4. D'Alessandro ME, Chicco A, Karabatas L, Lombardo YB: Role of skeletal muscle on impaired insulin sensitivity in rats fed a sucrose rich diet: Effect of moderate level of dietary fish oils. Nutrition 2000, 11(5):273-280.

5. Ohia SE, Opere CA, Stohs SJ: Safety and mechanism of appetite suppression by a novel hydroxyl citric acid extract. Mol cell biochem 2002, 338:89-103.

6. Roberts CK, Berger JJ, Barnard RJ: Long-term effects of diet on leptin, energy intake and activity in a model of diet-induced obesity. Appl Physio 2002, 93:887-893.

7. Trinder p: Determination of blood glucose using an oxidase-peroxidase system with a non-carcinogenic chromogen. J Clin Pathol 1969, 22(2):158-161.

8. Fawcett JK, Scott JE: A rapid and precise method for the determination of urea. $J$ clin path 1960, 13:156-159.

9. Henry RJ: Clinical Chemistry, Harper \& Row Publishers, New York; 1964, 181.

10. Glock GE, McLean P: Further studies on the properties and assay of glucose 6-phosphate dehydrogenase and 6-phosphogluconate dehydrogenase of rat liver. Biochem J 1953, 55(3):400-408.

11. Mihara M, Uchiyama M: Determination of malonaldehyde precursor in tissue by thiobarbituric acid test. Anal Biochem 1978, 86(1):271-278.

12. Cohen G, Dembiec D, Marcus J: Measurement of catalase activity in tissue extracts. Anal Biochem 1970, 34(1):30-38.

13. Miranda KM, Espey MG, Wink DA: A rapid, simple spectrophotometric method for simultaneous detection of nitrate and nitrite. Nitric Oxide 2001, 5(1):62-71

14. Sastre J, Pallardó FV, Llopis J, Furukawa T, Viña JR, Vina J: Glutathione depletetion by hyperphagia induced obesity. Life sci 1989, 45(2):183-187.

15. La fleur SE, VanRozen AJ, Groen weg E, Adan RA: A free choice high fat, high sugar diet induces changes in arcuate neuropeptids expression that support hyperphagia. Int J obes 2009, 22(4):55-63.

16. Savastano DM, Covasa M: Adaptation to a high-fat diet leads to hyperphagia and diminished sensitivity to cholecystokinin in rats. J Nutr 2005, 135(8):1953-1959.

17. Dziedzic B, Szemraj J, Bartkowiak J, Walczewska A: Various dietary fats differentially change the gene expression of neuropeptides involved in body weight regulation in rats. J Neuroendocrinol 2007, 19(5):364-373.

18. Akiyama T, Tachibana I, Shirohara H, Watanabe N, Otsuki M: High-fat hypercaloric diet induced obesity, glucose intolerance and hyperlipidemia in normal adult male wister rat. Diabetes Res \& clinical prac 1996, 31(1-3):27-35.

19. Lindqvist A, Baelmans A, Erlanson-Albertsson C: Effect of sucrose, glucose and fructose on peripheral and central appetite signals. Regul Pept 2008, 150(1-3):26-32.

20. Lomba A, Milagro Fl, García-Díaz DF, Campión J, Marzo F, Martínez JA: A high-sucrose isocaloric pair-fed model induces obesity and impairs NDUFB6 gene function in rat adipose tissue. J Nutrigenet Nutrigenomics 2009, 2(6):267-272

21. Mattes RD, Bormann L: Effects of (-)-hydroxycitric acid on appetitive variables. Physiol Behav 2000, 71(1-2):87-94.

22. Buettner R, Schölmerich J, Bollheimer LC: High-fat diets: modeling the metabolic disorders of human obesity in rodents. Obesity 2007, 15:798-808.
23. Kanazawa M, Xue CY, Kageyama H, Suzuki E, Ito R, Namba Y, Osaka T, Kimura S, Inoue S: Effects of a high-sucrose diet on body weight, plasma triglycerides, and stress tolerance. Nutr Rev 2003, 61(5-2):27-33.

24. Roberts CK, Barnard RJ, Vaziri ND: Oxidative stress and dysregulation of NADPH oxidase and antioxidant enzyme in diet induced metabolic syndrome. Metabolism 2006, 55(7):928-934.

25. Hamidian Jahromi A, Jalali GA, Roozbeh J: Impact of obesity on development of chronic renal allograft dysfunction. Saudi J kidney Dis transp/ 2009, 20(3):375-377

26. Dobrian AD, Schriver SD, Khraibi AA, Prewitt RL: Pioglitazone prevents hypertension and reduces oxidative stress in diet-induced obesity. Hypertension 2004, 43(1):48-56.

27. Matsushita K, Yasuda G, Shouda M, Umemura S: Evaluation of renal function and protein urea body mass health examination in young obese Japanese people. Clin Exp Nephrol 2009, 13(4):316-324.

28. Corpeli JE, Bakker SJ, Stoic RP: Obesity and impaired renal function potential for life style intervention. Eur J Epidemiol 2009, 24(6):275-280

29. Li Y, Qi Y, Kim MS, Xu KZ, Huang TH: Increased renal collagen cross linking and lipid accumulation in nephropathy of zucker diabetic fatty rats. Diabetes Metab Res 2008, 24(6):498-506

30. Altunkaynak ME, Ozbek E, Altunkaynak BZ, Can I, Unal D, Unal B: The effect of high fat diet on renal structure and morphometric parametric of kidney in rats. J Anat 2008, 212(6):845-852.

31. Prior LJ, Eikelis N, Armitage JA, Davern PJ, Burke SL, Montani JP, Barzel B, Head GA: Exposure to a high-fat diet alters leptin sensitivity and elevates renal sympathetic nerve activity and arterial pressure in rabbits. Hypertension 2010, 55(4):862-868.

32. Kashihara N, Satoh M: Molecular pathogenesis of chronic kidney disease. Nippon Rinsho 2008, 66(9):1267-7.

33. Knight SF, Imig JD: Obesity, insulin resistance and renal function. Microcirculation 2007, 14(4-5):349-362.

34. Asghar M, Monjok E, Kouamou G, Bagchi D, Lokhandwala MF: Super citric max (HCA-SX) attenuate increase in oxidative stress, inflammation, insulin resistance, body wight in developing obese zucker rats. $\mathrm{Mol}$ cell Biochem 2007, 304(1-2):93-99.

35. Fukuchi S, hamaguch K, Seike M, Himeno k, Skata T, Yoshimatsu H: Role of fatty acid composition in development of metabolic disorders in sucrose induces obese rats. Exp Boil Med 2004, 229(6):486-493.

36. Sarafidis PA, Ruilope LM: Insulin resistance, hyperinsulinemia and renal injury: mechanisms and implications. Am J Nephrol 2006, 26(3):232-244.

37. Kim KY, lee HN, Kim YJ, Palk T: Garcinia cambogia extract ameliorates visceral adiposity in C57BL/6 j mice fed on high fat diet. Biosci Biotechnol Biochem 2008, 72(7):1772-1780.

38. Xu Y, Osborne BW, Stanton RC: Diabetes causes inhibition of glucose-6phosphate dehydrogenase via activation of PKA, which contributes to oxidative stress in rat kidney cortex. Am J Physiol Renal Physiol 2005, 289(5):F1040-1047

39. Kletzien RF, Harris PK, Foellmi LA: Glucose 6 phD: a "house keeping" enzyme subject to tissue specific regulation by hormones, nutrient and oxidant stress. FASEB J 1994, 8:174-181.

40. Priego T, Sanchez J, Palou A, Pico C: Effect of HFD feeding on leptin receptor expression in white adipose tissue in rats: depot- and sexrelated differential response. Genes Nutr 2009, 4(2):151-156.

41. Naumnik B, Myśliwiec M: Renal consequences of obesity. Med Sci Monit 2010, 16(8):RA163-170.

42. Roy S, Shah H, Rink C, Khanna S, Bagchi D, Bagchi M, Sen CK: Transcriptom of primary adipocytes from obese woman in response to novel hydroxycitric acid- based dietary supplement. DNA cell Biol 2007, 26(9):627-639.

43. Saito M, Nagala J, Tokeuchi M: High dose of garcinia is effective in suppressing fat accumulation in developing male zucker obese rats but highly toxic to testes. Food Chem Toxicol 2005, 43(3):411-419.

44. Bloch K, Shichman E, Vorobeychik M, Bloch D, Vardi P: Catalase expression in pancreatic alpha of diabetic and non diabetic mice. Histochem cell Biol 2007, 127(2):227-232

45. Chinen I, Shimabukuro M, Yamakawa K, Higa N, Matsuzaki T, Ueda S, Sakanashi M, Takasu N: Vascular lipotoxicity: endothelial dysfunction via fatty-acid-induced reactive oxygen species overproduction in obese Zucker diabetic fatty rats. Endocrinology 2007, 148:160-165. 
46. Aljofan $M$, Ding $H$ : High glucose increase expression of cyclooxogenase 2 , increase oxidative stress and decrease the generation of nitric oxide in mouse microvessel endothelial cells. J cell physiol 2010, 222(3):669-675.

47. Martins MA, Catta-Preta M, Mandarim-de-Lacerda CA, Aguila MB, Brunini TC, Mendes-Ribeiro AC: High fat diets modulate nitric oxide biosynthesis and antioxidant defence in red blood cells from C57BL/6 mice. Arch Biochem Biophys 2010, 499(1-2):56-61.

48. Payne GA, Bratz IN, Dick GM, Tune J D: Endogenus adipose derived factor diminish coronary endothelial function via inhibition of nitric acid synthase. Micro Circulation 2008, 15(5):417-426.

49. Canabal DD, Potian JG, Duran RG, McArdle JJ, Routh VH: Hyperglycemia impairs glucose and insulin regulation of nitric oxide production in glucose-inhibited neurons in the ventromedial hypothalamus. Am J Physiol Regul Integr Comp Physiol 2007, 293(2):R592-600.

50. Morrison RG, Mills C, Moran AL, Walton CE, Sadek MH, Mangiarua El, Wehner PS, McCumbee WD: A moderately high fat diet promotes saltsensitive hypertension in obese zucker rats by impairing nitric oxide production. Clin Exp Hypertens 2007, 29(6):369-381.

51. Claudino MA, Franco-penteado CF, Camarago EA, Teixdeira SA: Up regulation of $\mathrm{Gp} 91$ (phox) subunit of $\mathrm{NAD}(\mathrm{P}) \mathrm{H}$ oxidase cotribute to erectile dysfunction caused by long term nitric oxide inhibition in rats: reversion by regular physical training. Urology 2009, 22:44-55.

doi:10.1186/1476-511X-10-6

Cite this article as: Amin et al:: Protective effect of Garcinia against renal oxidative stress and biomarkers induced by high fat and sucrose diet. Lipids in Health and Disease 2011 10:6.

\section{Submit your next manuscript to BioMed Central and take full advantage of:}

- Convenient online submission

- Thorough peer review

- No space constraints or color figure charges

- Immediate publication on acceptance

- Inclusion in PubMed, CAS, Scopus and Google Scholar

- Research which is freely available for redistribution

Submit your manuscript at www.biomedcentral.com/submit 